\title{
X-Ray Grazing Incidence Diffraction from Alkylsiloxane Monolayers on Silicon Wafers
}

\section{Citation}

Tidswell, I. M., T. A. Rabedeau, Peter S. Pershan, S. D. Kosowsky, J. P. Folkers, and George M. Whitesides. 1991. X-ray grazing incidence diffraction from alkylsiloxane monolayers on silicon wafers. Journal of Chemical Physics 95(4): 2854-2861.

\section{Published Version}

doi:10.1063/1.460886

\section{Permanent link}

http://nrs.harvard.edu/urn-3:HUL.InstRepos:10357545

\section{Terms of Use}

This article was downloaded from Harvard University's DASH repository, and is made available under the terms and conditions applicable to Other Posted Material, as set forth at http:// nrs.harvard.edu/urn-3:HUL.InstRepos:dash.current.terms-of-use\#LAA

\section{Share Your Story}

The Harvard community has made this article openly available.

Please share how this access benefits you. Submit a story.

Accessibility 


\title{
X-ray grazing incidence diffraction from alkylsiloxane monolayers on silicon wafers
}

\author{
I. M. Tidswell, T. A. Rabedeau, P. S. Pershan, and S. D. Kosowsky \\ Department of Physics and Division of Applied Sciences, Harvard University, Cambridge, \\ Massachusetts 02138
}

J. P. Folkers and George M. Whitesides

Department of Chemistry, Harvard University, Cambridge, Massachusetts 02138

(Received 19 March 1991; accepted 3 May 1991)

\begin{abstract}
$\mathrm{X}$-ray reflection (both specular and off-specular) and grazing incidence diffraction (GID) have been used to study the structure of alkylsiloxane monolayers $\left(n-\mathrm{C}_{18} \mathrm{H}_{37} \mathrm{SiO}_{1.5}\right)$ formed by self-assembly from solution on silicon wafers. GID studies of complete monolayers reveal a single ring of scattering associated with the monolayer. The Lorentzian line shape of this ring indicates that the film is characterized by liquidlike order, with a typical translational correlation length of about $45 \AA$. The thermal coefficient of expansion of the monolayer, as determined from the GID peak position, is approximately equal to the value for liquid $n$ alkanes. Upon either heating or cooling, the monolayer correlation lengths decrease, suggesting that the differential thermal-expansion coefficients of the film and substrate figure prominently in thermal changes of the molecular ordering. GID data for incomplete monolayers also reveal a single ring of scattering associated with the monolayer. While both the translational correlation lengths and integrated peak areas are significantly reduced relative to complete monolayers, the peak positions of the incomplete monolayers are comparable to those of complete monolayers. Given the lower average areal density of incomplete monolayers, this finding implies that incomplete monolayers are inhomogeneous.
\end{abstract}

\section{INTRODUCTION}

Monolayers composed of hydrocarbon chains are interesting both for their similarity to biological membranes and for their technological potential. ${ }^{1}$ For example, alkylsiloxane monolayers that contain dipolar functional groups have been used to fabricate nonlinear optical devices ${ }^{2}$ and similar monolayers may find applications in techniques for the fabrication of quantum-well devices. ${ }^{3}$ In addition, alkylsiloxane monolayers are used for a wide variety of applications such as adhesion promoters ${ }^{4}$ and surface protective films. ${ }^{1}$ Although hydrocarbon monolayers have been the subject of many studies (see below), characterization of their in-plane structural order remains incomplete.

With the development of synchrotron $x$-ray sources, it is possible to conduct grazing incidence diffraction (GID) studies of long-range molecular order of the two-dimensional (2D) structure of monolayers on both liquid and solid substrates. On the basis of numerous theoretical efforts (for a review, see Ref. 5) it is now understood that, as the result of either thermal or quantum-mechanical zero-point fluctuations, the commonly accepted 2D-"crystalline" phase is not strictly periodic. Nevertheless, since deviations from strict periodicity change quite slowly with molecular separation (varying logarithmically with distance) the effect is only detected in the most delicate experiments. ${ }^{6}$ It is convenient to neglect this detail in discussing the type of order that has been observed experimentally in this study. A second theoretical result that has been confirmed experimentally and which we cannot ignore is that there exists a hexatic phase, ${ }^{5}$ in which the molecules have short-range transla- tional order (i.e., liquidlike) and long-range orientational order in the positions of the nearest neighbors. For example, consider crystalline regions that are in registry with a hypothetical underlying lattice. While rotation of one of these regions destroys both the rotational and translational order, translation of one region without rotation destroys only the translational order.

GID studies of Langmuir films at the water/vapor interface ${ }^{7-10}$ have revealed resolution-limited Bragg-like diffraction peaks that are indicative of $2 \mathrm{D}$ phases with relatively long-range translational order; typical half-widths imply domain sizes in excess of $500 \AA$ that are probably crystalline. The structure of these crystalline phases depends sensitively on the molecular area, temperature, and specific surfactant molecule. Similarly, a GID study of a phospholipid monolayer deposited on the surface of a single crystal of silicon by the Langmuir-Blodgett (LB) technique ${ }^{11}$ indicated that the monolayer contained regions in which long-range translational order extended over long length scales $\gtrsim 5000 \AA \AA$ and the orientational order extended over $\sim 10000 \AA$. In addition, the study revealed that a reversible structural transition occurred at about $65^{\circ} \mathrm{C}$, for which the intensity of the peak observed in GID was significantly reduced, although the peak half-width at half-maximum (HWHM) remained unchanged.

A transmission electron microscopy (TEM) study of cadmium stearate LB monolayer films on silicon wafers ${ }^{12}$ indicated that the molecules had a molecular area of $21 \AA^{2}$ and were arranged in an undistorted hexagonal structure with very-long-range orientational order $(>1 \mathrm{~mm})$ and 
short-range liquidlike translational order of about $40 \AA$ (i.e., hexatic order). A low-energy electron diffraction (LEED) investigation of cadmium stearate films on noble-metal substrates $^{13}$ gave similar results with a reported translational correlation length of about $140 \AA$. Another TEM study of cadmium stearate, arachidate, and behenate (Cd $\left[\mathrm{OOC}\left(\mathrm{CH}_{2}\right)_{n} \mathrm{CH}_{3}\right]$ where $n=16,18$, and 20, respectively) LB monolayers on silicon-oxide substrates ${ }^{14}$ again suggested long-range orientational order and short-range translational order (correlation lengths $85-130 \AA$ ). Upon heating, all the samples were found to undergo an irreversible loss of peak intensity (e.g., falling to zero at about $95^{\circ} \mathrm{C}$ for cadmium behenate). This loss of signal was interpreted as resulting from the loss of a small fraction of the molecules from the surface and consequent increase of the overall random disorder. While the peak could be measured, the translational and orientational correlation lengths were essentially independent of temperature.

A study of self-assembled organosulfur compounds with a single hydrocarbon chain on gold single crystals using $(T E M)^{15}$ indicated that the molecular area was again about $21 \AA^{2}$ with short-range-like translational order (about 60 $200 \AA$ ) and much longer-range orientational order. Details of the molecular area, translational and orientational order, chain packing density, and unit cell were found to be dependent on the crystallographic axes of the gold face. A helium diffraction measurement of a similar system ${ }^{16}$ at temperatures below $50 \mathrm{~K}$ again found only relatively short-range translational order $(<100 \AA)$, which decreased rapidly with increasing temperature. Finally, a recent GID study of a room temperature 18 carbon chain alkylsiloxane monolayer self-assembled onto a silicon wafer ${ }^{17}$ found one peak indicative of a molecular area of approximately $19.5 \AA^{2}$. The peak was broad, indicating a translational correlation length of about $25 \AA$.

The morphology of hydrocarbon chain monolayers has been studied theoretically in several molecular-dynamics (MD) calculations in which the head group was either free to move in the plane of the surface, as is the case for Langmuir films on water, ${ }^{18-21}$ or fixed at specific lattice sites, as found for alkanethiol monolayers on gold. ${ }^{22}$ These calculations provide explicit predictions of the atomic positions, from which scattering cross sections have been calculated. Typically, the calculated in-plane translational correlation lengths are quite short; this result and the mean molecular tilt for a given mean molecular area ${ }^{21}$ are both in agreement with experimental results. ${ }^{8}$ The small system sizes used in these calculations, however, cast some doubt on the signifcance of this agreement between theory and experiment.

In this paper we report measurement of the $x$-ray specular and off-specular reflection and GID scattering from fully formed and incomplete ( $>50 \%$ complete) 18 carbon alkylsiloxane monolayers self-assembled from solution on silicon wafers. The x-ray data allow characterization of the in-plane translational order, correlation lengths, and the molecular packing density. The evolution of the in-plane structure over the temperature range $0-100^{\circ} \mathrm{C}$ is also detailed. Finally, a brief discussion of the susceptibility of the monolayers to $\mathrm{x}$ ray damage is presented.

\section{PREPARATION OF MONOLAYERS}

The monolayers were formed by self-assembly from solution on polished $\mathrm{Si}(100)$ wafers (Semiconductor Processing Co., Boston, MA) with dimensions of approximately 35 $\mathrm{mm} \times 20 \mathrm{~mm}$. Previous studies ${ }^{23}$ have shown that these wafers are coated with a native silicon-oxide film about $15 \AA$ thick, the surface of which has a rms roughness of about 2.5$3.5 \AA$ A.

Preparation of the monolayers has been described previously. ${ }^{23,24}$ Here, we present only an outline of the method followed and indicate variations from the previous procedure. Initially, the wafers were cleaned in a strongly oxidizing mixture of sulfuric acid and hydrogen peroxide to remove all carbonaceous material. Wafers were then rinsed in copious amounts of deionized water and used within $15 \mathrm{~min}$ of removal from the oxidizing solution.

Monolayers were formed by self-assembly from dilute solutions of octadecyltrichlorosilane $\left(n-\mathrm{C}_{18} \mathrm{H}_{37} \mathrm{SiCl}_{3}\right.$, OTS) in hexadecane in a glovebox with low relative humidity $(<10 \%)$. To form complete monolayers, the wafers were left in solution for two days. Partial monolayers were formed by immersing the samples in solution for shorter lengths of time; specifically, coverages of up to $80 \%$ required 15-60 min immersions while coverages of over $80 \%$ required up to a day in solution. Formation times for incomplete monolayers varied substantially and were strongly solution dependent. After removal from solution, all wafers were rinsed with methylene chloride, rinsed in ethanol, and blown dry in a stream of nitrogen.

Preliminary characterization of the monolayers was performed using ellipsometry ${ }^{25}$ and contact angle measurements. Table I lists the ellipsometrically determined film thicknesses and contact angles for water and hexadecane. The characteristics of the full monolayers (samples 1-3) are in agreement with those of previous studies. ${ }^{24,26}$ Experience over several years of study has found only small intersample variations for the full monolayers (the variations in Table I for the first three samples listed are typical); incomplete monolayers, however, are much more inhomogeneous, with larger variations between different regions of the same sample and between samples made from different solutions. The contact angles for the incomplete monolayers listed in Table $\mathrm{I}$ are similar to values measured in a previous study. ${ }^{27,28}$

TABLE I. The ellipsometric thickness and the advancing and receding contact angles (in degrees) of water and hexadecane (HD) for the five samples reported in this study. Samples 1-3 are "complete" monolayers whereas samples 4 and 5 are approximately $73 \%$ and $56 \%$ complete, respectively (as estimated based on their thickness, $l$ ). The uncertainties associated with these values are approximately $\pm 1 \AA$ for the ellipsometric length, $\pm 2^{\circ}$ for the water contact angles, and $\pm 1^{\circ}$ for the hexadecane contact angles.

\begin{tabular}{cccccc}
\hline \hline Sample & $l(\AA \AA \AA)$ & $\mathrm{H}_{2} \mathrm{O}$ adv & $\mathrm{H}_{2} \mathrm{O}$ rec & HD adv & HD rec \\
\hline 1 & 27.8 & 112 & 104 & 45 & 44 \\
2 & 27.5 & 112 & 105 & 45 & 45 \\
3 & 26.8 & 111 & 106 & 44 & 44 \\
4 & 19.8 & 103 & 86 & 44 & 41 \\
5 & 15.4 & 101 & 85 & 43 & 39 \\
\hline
\end{tabular}




\section{X-RAY MEASUREMENTS}

GID is a powerful tool for studying the in-plane structure of thin films. ${ }^{7-10,29}$ It utilizes the fact that $x$ rays incident below a critical angle $\alpha_{c}$ are subject to total external reflection; $\alpha_{c} \approx \sqrt{2 \delta} \approx \lambda\left(\rho r_{0} / \pi\right)^{1 / 2}$, the refractive index is $n=1-\delta+i \beta, \rho$ is the substrate electron density, and $r_{0}$ the classical electron radius. For most condensed matter, the critical angle lies between $0.1^{\circ}$ and $0.5^{\circ}$ for $x$ rays of wavelength $1-2 \AA$. The electric fields associated with $x$ rays incident at angles smaller than $\alpha_{c}$ decay exponentially in the substrate with typical penetration lengths of order 50-100 $\AA$. When the incident beam strikes the surface at the critical angle, boundary conditions requiring the continuity of the electric field across the interface result in a surface field with twice the amplitude of the incident field. For angles smaller than the critical angle, the surface fields fall monotonically to zero at $0^{\circ}$; for angles larger than the critical angle, this field falls rapidly to the value of the incident field. A similar enhancement of the field of the detected wave occurs when the outgoing angle (i.e., the angle a photon scattered into the detector makes with respect to the surface plane) is at or near the critical angle. In principle, setting both the incident and outgoing angles to the critical angle leads to an enhancement of surface scattering by a factor of 16 (ignoring absorption effects); however, in practice enhancements of 3-10 are achieved. An additional advantage of using the GID technique is that for incident angles less than $\alpha_{c}$, exclusion of the electric field from the bulk material significantly reduces extraneous diffuse scattering from the bulk.

$\mathrm{X}$-ray specular reflectivity is complementary to GID, probing surface and interface structure along the surface normal direction. For sufficiently smooth interfaces with low- $Z$ materials, ${ }^{30}$ the reflectivity is described by ${ }^{23,31}$

$$
R\left(q_{z}\right)=R_{F}\left(q_{z}\right)\left|\Phi\left(q_{z}\right)\right|^{2},
$$

with

$$
\Phi\left(q_{z}\right)=\int \frac{1}{\rho_{\infty}} \frac{d\langle\rho\rangle}{d z} e^{i q z} d z,
$$

where $R_{F}$ is the theoretical Fresnel reflectivity from an ideally sharp smooth interface, $q_{z}=(4 \pi / \lambda) \sin (\theta), \theta$ is the angle between the incident beam and the sample surface, $d\langle\rho\rangle / d z$ is the derivative of the electron-density profile averaged over the in-plane coherence length of the $\mathrm{x}$ rays, and $\rho_{\infty}$ is the electron density of the semi-infinite bulk. ${ }^{23}$ This form is valid for $\theta$ larger than approximately twice $\alpha_{c}$. Previous x-ray reflectivity measurements of these monolayers ${ }^{23}$ have shown that $d\langle\rho\rangle / d z$ is uniform over in-plane length scales longer than about $10^{4} \AA$ and that the molecules have a mean molecular area of $22.5 \pm 2.5 \AA^{2}$. These results imply either that the chains are extended to about $90 \% \pm 10 \%$ of their full length or that the monolayers are $90 \% \pm 10 \% \mathrm{com}$ plete.

In this study measurements were performed on the AT\&T beam line X16B at the National Synchrotron Light Source using a wavelength of $1.691 \AA$. The beam line was equipped with a bent mirror focusing in the vertical plane, and an asymmetrically cut, bent $\mathrm{Ge}(111)$ crystal monochromator which focused in the horizontal plane. The spectrom- eter was configured for scattering in the vertical plane. The incident beam was tightly focused and, for the GID studies, essentially all of the beam was passed through slits of dimension $1.0 \mathrm{~mm}$ wide by $1.0 \mathrm{~mm}$ high, positioned approximately $300 \mathrm{~mm}$ before the sample. With these slit dimensions the incident beam "footprint" was larger than the sample size when the sample was positioned at or below the critical wave vector $\left[q_{\mathrm{z}}=q_{\mathrm{c}} \approx 0.0313 \AA^{-1}\right.$ for silicon, where $q_{c}=(4 \pi / \lambda) \sin (\alpha)$ and $\alpha$ is the angle the incident beam makes with the surface ]. The detector arm slits were $8.0 \mathrm{~mm}$ wide by $2.5 \mathrm{~mm}$ high on a $660 \mathrm{~mm}$ long arm, giving an inplane longitudinal resolution of about $0.014 \AA^{-1}$. For the scattering angles of interest, the projected area of the detector slits at the sample position was smaller than the sample size.

Samples were mounted inside a cell made from a Rubbermaid No. 8 food storage container, with the $x$ rays incident on the sample through 0.002 in. thick Kapton windows cemented over holes in the container walls. A slow, continuous flow of helium was maintained through the cell. The samples were mounted on a plate attached to a thermoelectric device that was used to heat and cool the samples over the temperature range of $0-100^{\circ} \mathrm{C}$.

Reflectivity data for these samples were taken using the same spectrometer configuration with incident slits $0.05 \mathrm{~mm}$ high by $1 \mathrm{~mm}$ wide for incident angles less than $2^{\circ}$, and 0.3 $\mathrm{mm}$ high by $1 \mathrm{~mm}$ wide for larger incident angles. These dimensions restricted the beam to fall wholly into the sample for all incident angles except those much smaller than the critical angle. The detector slits were set to $1 \mathrm{~mm}$ high by 4 $\mathrm{mm}$ wide for all reflectivity measurements. Small-angle reflectivity measurements were made using attenuators to avoid saturating the detector.

The alkylsiloxane GID peak was found to be quite sensitive to $\mathrm{x}$-ray induced damage; the $\mathrm{x}$-ray reflectivity was somewhat less sensitive. Previous studies ${ }^{32}$ have shown that the presence of atmospheric oxygen during $x$-ray exposure increases damage to the monolayer. To mitigate these effects, the samples were maintained in a helium atmosphere and were periodically translated tangentially to the beam so that no section of a sample was exposed to the beam for a period of more than $1 \mathrm{~h}$; typically exposure was limited to 30 $\min$.

\section{RESULTS AND DISCUSSION}

\section{A. Complete monolayers}

Figure 1(a) shows the GID structure observed from a clean silicon wafer and from a wafer coated with an alkylsiloxane monolayer. In order to avoid the Bragg peaks from the silicon substrate, both scans were taken along the bulk silicon $(h, 2 h)$ direction with a $q_{z}$ component of $0.025 \AA^{-1}$, slightly less than the silicon substrate critical angle. The only feature associated with the alkylsiloxane monolayer is the powderlike diffraction ring at $1.5 \AA^{-1}$. The intensity of this ring was independent of the direction of the radial scan. No higher-order peaks associated with the alkylsiloxane monolayer were observed on any samples. Figure 1 (b) shows the structure of the GID peak for a different region of the same 


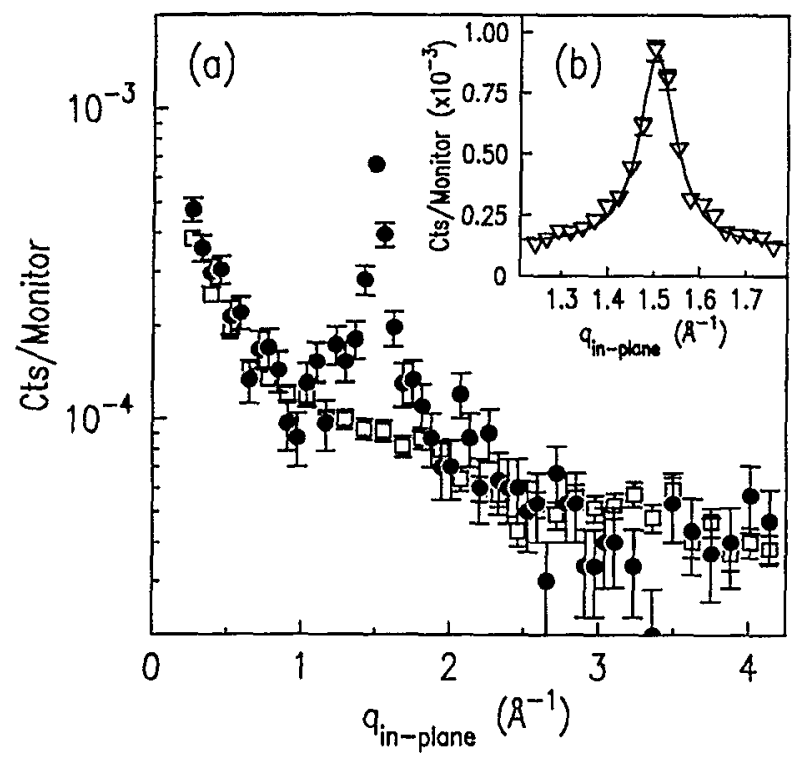

FIG. 1. Shown in (a) is the comparison of GID scans for uncoated ( $\square$ ) and $\mathrm{C}_{1}$ alkylsiloxane coated $(\bullet)$ silicon wafers. Shown in greater detail in (b) is the peak at $1.5 \AA^{-1}$ for a different region of the same sample, together with a fit to the data using a Lorentzian peak shape on a linearly sloping background.

sample in more detail, together with a fit to the peak using a Lorentzian curve on a linearly sloping background. Typically, a Lorentzian peak shape gave fits to the data with $\chi^{2}$ values between 1 and 1.5; a Gaussian model of the peak shape was found to give fits with typical $\chi^{2}$ values of above 4 . The GID peak is quite broad, having a HWHM $(\Delta q / 2)$ of $0.022 \AA^{-1}$. Correcting for the slight broadening of the peak by the spectrometer resolution, this corresponds to an inplane molecular correlation length, $2 / \Delta q$, of about $45 \AA$, or about 9 molecular diameters. In view of the fact that the liquid state is typically characterized by an exponential decay of order in real space (Lorentzian in reciprocal space), whereas a fine powder of microcrystallites results in diffraction peak shapes more Gaussian than Lorentzian, the combination of the observed Lorentzian line shape and short translational correlation length suggests that the in-plane order of these monolayers is more liquidlike than crystalline.

Similar scans taken at various fixed $q_{z} \leqslant 0.46 \AA^{-1}$ reveal that the intensity of the peak falls rapidly just above the critical angle and much more slowly for $q_{z} \gtrsim 2 q_{c}$; for $q_{z} \geqslant 0.30$ $\AA^{-1}$ no peak was observed. The data suggest that the peak position shifted to slightly lower $q_{\text {in-plane }}$ with increasing $q_{z}$, although the broad peak width and low intensity do not allow for a quantitative measurement. The existence of only one scattering peak suggests that the local order is simple, undistorted hexagonal. In particular, the absence of any peaks for $0.3 \AA^{-1} \leqslant q_{z} \leqslant 0.46 \AA^{-1}$ coupled with the $\sim 21^{\circ}$ maximum possible average molecular tilt (determined from specular reflectivity data; see below) tends to exclude the possibility that the chains are locally tilted with order like that of the smectic-I liquid-crystal phase ${ }^{33}$ such as is observed for Langmuir monolayers on water. ${ }^{10}$ Smectic-F or- der is also excluded since only one in-plane diffraction peak was observed.

The specular reflection data from a typical alkylsiloxane monolayer (sample 3 of Table 1) is shown in Fig. 2(a). The Fresnel normalized reflectivity [Fig. 2(b)] was modeled using the same structure as in a previous study, ${ }^{23}$ namely a four-layer model [the layers representing the hydrocarbon, interfacial (head-group) region, silicon-oxide and semi-infinite silicon layers] with the layers separated by interfaces with error function form (i.e., $d\langle\rho\rangle / d z$ has a Gaussian profile). The best fit to the data using this model is shown as the solid line, with the real-space model it represents shown in Fig. 2(c). The length of the hydrocarbon film (excluding the silicon head group), extracted using the same construction as a previous study, was found to $\mathrm{be}^{23} 21.3 \pm 0.5 \AA$. In addition, helium/hydrocarbon and silicon-oxide/silicon interfaces had rms widths of approximately 2.5 and $2.0 \AA$, respectively, and the hydrocarbon/head-group/silicon-oxide region had a combined rms width of about $4.5 \AA$. These parameters are almost identical to those determined in a previous study. ${ }^{23,34}$ The widths of the hydrocarbon interfaces and the construction used to estimate the thickness, however, may introduce systematic errors, raising the possibility that the thickness uncertainty is underestimated. A mono-

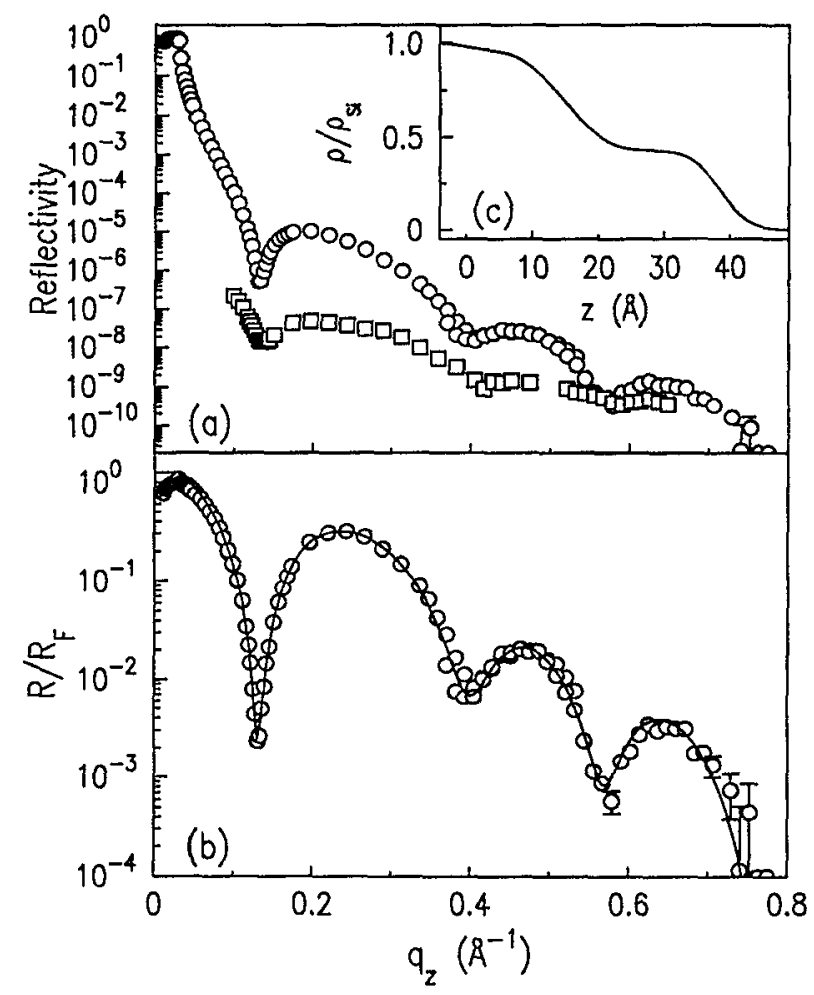

FIG. 2. Shown in (a) is the specular reffectivity profile for an alkylsiloxane coated surface $(O)$. The squares show the diffuse scattering from the same sample, taken along a direction $q_{x}=q_{z} / 500$. Shown in (b) is the Fresnel normalized reflectivity, together with best fit $(-)$ to the model described in the text. The real-space electron-density profile represented by this fit is shown in (c). 
layer thickness of $21.3 \pm 0.5 \AA$ leads to an average length per methylene group of $1.18 \pm 0.03 \AA$. This compares to the length of $22.7 \AA$ expected for a fully extended chain with a length per methylene group of $1.265 \AA$. The slightly smaller thickness of the monolayers compared to that expected for fully extended chains can be associated with either a mean molecular tilt of $21 \pm 4^{\circ}$ or to some concentration of gauche bonds in the hydrocarbon chains.

Using the values for the electron density, $\rho_{e}$, and the average length of the molecule, $L$, obtained from the fits to the normalized specular reflection data, the average molecular area of the alkylsiloxane monolayers was calculated to be $N_{e} /\left(L \rho_{e}\right)=22.3 \pm 1.6 \AA^{2}$. This value is consistent with the results of previous reflectivity studies ${ }^{23}$ of $22.5 \pm 2.5 \AA^{2}$. For comparison, the position of the GID peak indicates a molecular area of $20.2 \pm 0.2 \AA^{2}$ (assuming a simple hexagonal packing), similar to the areas (19.4-21.4 $\AA^{2}$ ) reported in other studies of hydrocarbon monolayers on solid substrates. ${ }^{12,13,15-17}$ Since the discrepancy between the areas determined in this study by GID and reflectivity is only slightly larger than the limits that are determined by the quoted errors, the data are only slightly indicative of monolayer inhomogeneity.

Also shown in Fig. 2(a) is the diffuse scattering taken along a direction with $q_{x}=q_{z} / 500$, which corresponds to a scan with $2 \theta$ set approximately $0.25^{\circ}$ from the specular condition, well away from the specular beam. The presence of oscillations in the diffuse scattering along $q_{z}$ indicate that the roughness of the hydrocarbon/air interface is correlated with the roughness of the underlying hydrocarbon/siliconoxide interface, the diffuse scattering from the two interfaces interfering. This interference indicates the monolayer film has a roughly constant thickness, which follows the surface undulations of the hydrocarbon/silicon-oxide interface.

The combination of both specular and GID measurements presents a picture of the monolayer as a structure with in-plane liquidlike local order (that is, short-range exponential decay of real-space translational correlations in the plane of the film). While the measured translational correlation lengths are about $45 \AA$, it is not established whether there exist more disordered regions that have a lower electron density and do not contribute to the GID peak. An attempt to visualize a one-dimensional projection of this order is shown in Fig. 3. The thermal response of the GID peak (see later) indicates that the region close to the silicon-oxide does not scatter significantly into the GID peak. In addition, the tail of the hydrocarbon film is expected to be more disordered than the main body of the chains. Since we have no information on the order near the head groups or on the chain conformation near the alkane chain surface, these regions have been obscured in Fig. 3 to avoid presenting an erroneous picture. Scattering data indicate that the mean thickness of the complete monolayers is approximately $93 \%$ of the length calculated for fully extended chains (i.e., none of the "fully formed" monolayers in fact consist of fully extended molecules) and the local molecular packing is undistorted hexagonal. Measurements of the diffuse scattering indicate that the monolayer follows the surface undulations, with the chains forming a film of approximately constant thickness.

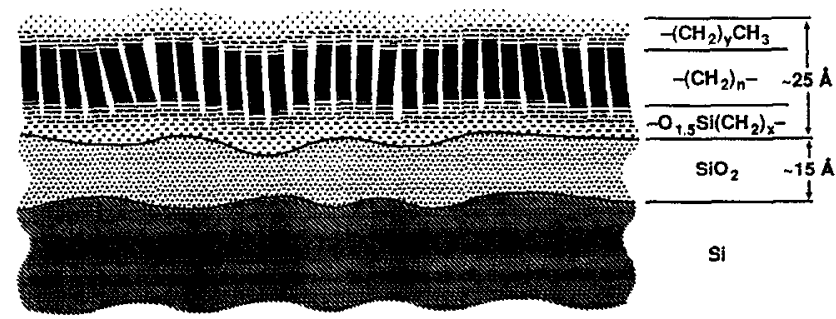

FIG. 3. Representation of the possible in-plane chain order projected onto one dimension, as determined from the width and shape of the GID peak. From the $\mathrm{x}$-ray data we are not able to determine the monolayer order near the headgroup $\left[-\mathrm{O}_{1,5} \mathrm{Si}\left(\mathrm{CH}_{2}\right)_{x}-\right]$ or the order near the surface $\left[-\left(\mathrm{CH}_{2}\right)_{y} \mathrm{CH}_{3}\right]$. To avoid presenting an erroneous picture, the order in the head and tail regions has been obscured. We show the order extending over some region of the molecules approximately in the center of the monolayer $\left[-\left(\mathrm{CH}_{2}\right)_{n}-\right]$ where $n=17-x-y$.

\section{B. Incomplete monolayers}

Figure 4 shows the variations of the GID peak between different "complete" and "incomplete" samples. Focusing first on the "complete" alkylsiloxane films (those with hydrocarbon film thickness greater than $21 \AA$ ), variations of

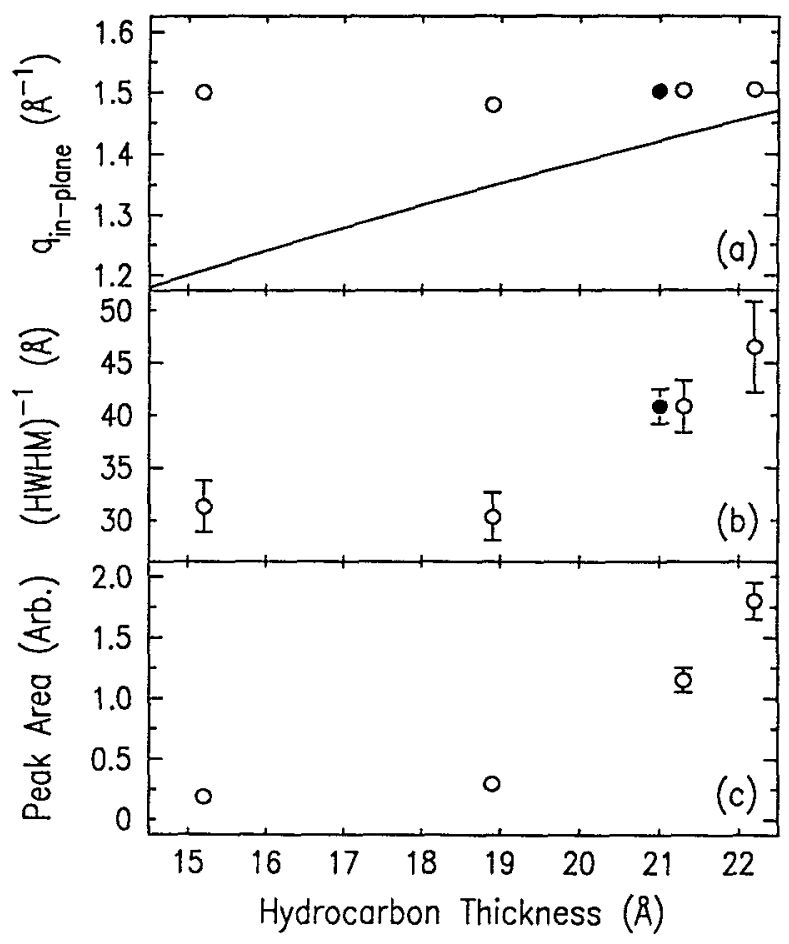

FIG. 4. Structural features of the alkylsiloxane films from the GID peak. Shown as a function of the hydrocarbon film thickness (as determined from using the technique described in Ref. 23) are (a) the position of the GID peak, (b) the coherence length [GID peak (HWHM) ${ }^{-1}$ ], and (c) the integrated area of the GID peak. The line in (a) represents the expected position of the GID peak from the molecular area as determined by specular reflection (see text for details). The data point shown as the solid circle was taken on a different beam line from the other data and hence the integrated area of this peak could not be compared to the other data. The error bars in this figure and Fig. 5 indicate the typical variations found across the sample and not the measurement uncertainty of one measurement of one region of the sample, which is in all cases much smaller. 
the monolayer thickness of less than $1.5 \AA$ correlate with large changes $(\approx 30 \%)$ in the integrated area of the peak while the position of the peak (and consequently the molecular area) are invariant. The solid line in Fig. 4(a) is the "expected" position of the GID peak if the mean separation between chains increased as the film thickness decreased.

Figure 4 also shows the intersample variations for films with much lower coverages of alkylsiloxane. The position of the GID peak is not a function of the alkylsiloxane mean molecular area, clear evidence that, for incomplete monolayers at least, the in-plane order is inhomogeneous. X-ray reflectivity measurements confirm an earlier result that the incomplete films have a reduced thickness and do not consist of islands of molecules surrounded by regions of zero or low coverage. ${ }^{23}$ The hydrocarbon/air interface, however, is broader for incomplete films when compared to full monolayers, again indicating some inhomogeneity over lengths less than the specular reflectivity in-plane coherence length of the $\mathrm{x}$ rays (about $10^{4} \AA$ ).

Although the GID peak position remains constant for incomplete monolayers, the in-plane order of such regions on the surface decreases as the mean alkylsiloxane coverage decreases. For less complete films the GID peak integrated intensity falls rapidly, with the scattering reduced by a factor of about 8 for a $56 \%$ complete film. It is interesting to note that as the molecular coverage decreases the in-plane translational correlation lengths also decrease, but more slowly than the integrated peak intensity, falling to about $30 \AA$ for a $56 \%$ film, equivalent to about six molecular diameters. The inter-and intrasample variations for incomplete monolayers were much larger than for complete monolayers. This observation is in agreement with wetting and reflectivity studies, which indicate that the process of forming incomplete monolayers is not as well controlled as for complete monolayers. ${ }^{24}$

\section{Thermal effects}

Figure 5 shows the temperature variation of the GID peak for a fully formed alkylsiloxane film. The specular reflectivity profile of the alkylsiloxane sample was measured before the temperature cycle began. This profile showed the sample to be a typical monolayer, with a hydrocarbon thickness of $21.0 \AA$. Figure 5(a) shows the variation in the GID peak position with temperature. These shifts are linear with temperature and quasireversible. From this figure the linear coefficient of thermal expansion of the monolayer was calculated to be $5.2 \times 10^{-4} \pm 0.5 \times 10^{-4} \mathrm{~K}^{-1}$. This value can be compared to a value of $5.1 \times 10^{-4} \mathrm{~K}^{-1}$ for liquid pentane, $3.0 \times 10^{-4}$ for liquid hexadecane, ${ }^{35}$ and $3 \times 10^{-6} \mathrm{~K}^{-1}$ for the silicon substrate, ${ }^{36}$ all at similar temperatures. The regions of the monolayer contributing to the GID peak have a thermal coefficient of expansion similar to thoses found for bulk $n$-alkanes. Curiously, the expansion coefficient is closer to the value found for short hydrocarbon chains $(n=5)$ than to the value for chains of length comparable to the monolayer chain length. Evidently, the portion of the molecular chain that yields the in-plane scattering peak thermally responds relatively independently of the tethered molecular

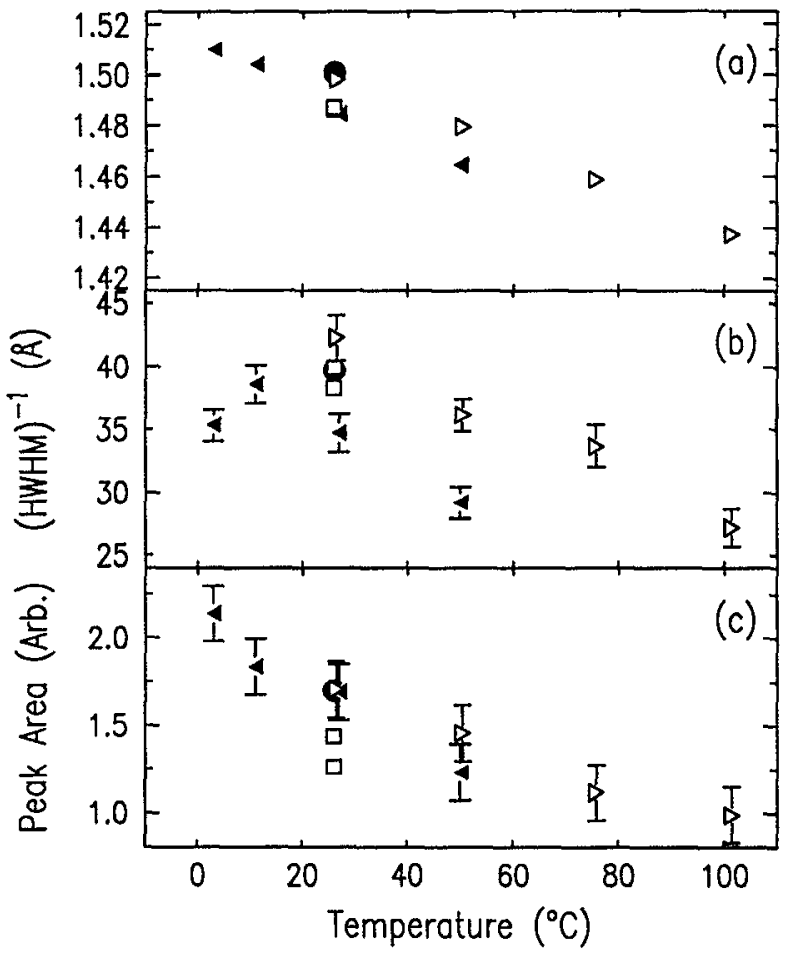

FIG. 5. Structural changes to one film on temperature changes. The symbols represent the order of the temperature cycle. Starting at room temperature $(O)$, the sample was first cooled $(\Delta)$, then heated $(D)$, then cooled again ( 4 ) back to room temperature ( $\square$ ). The GID peak position (a), coherence length (b), and integrated peak area (c) are shown for various temperatures. Each measurement was made on a fresh section of the sample to mitigate the effects of $\mathrm{x}$-ray damage. Error bars on some room-temperature measurements are omitted for clarity.

head group. A previous study of thermal effects on alkylsiloxane monolayers using $x$-ray specular reflectivity ${ }^{37}$ found no change to the film thickness upon heating or cooling and only small changes to the interface widths. In the present study the reflectivity profile of a monolayer sample measured after heating was also very similar to an unheated sample. These results indicate that moderate heating of an alkylsiloxane monolayer does not eject a significant number of molecules from the monolayer $(<1 \%)$.

The order resulting in the GID peak decreases with increasing temperature, as shown by the decrease in peak integrated area with increasing temperature [Fig. 5(c)]. Any change of temperature away from room temperature, however, increased the width of the GID peak, indicating a reduced translational correlation length [Fig. 5(b)]. This reduction of the in-plane coherence length for any change in temperature from room temperature is probably a consequence of packing frustration between different sections of the hydrocarbon molecules. The tail region of the hydrocarbon chains are free to expand in a manner similar to bulk hydrocarbons, whereas the head groups, which are firmly anchored to the silicon wafer, have a much lower thermal expansion coefficient imposed by the substrate. Using the measured thermal coefficient of expansion, we can check this hypothesis by calculating the movement between two 
molecules separated by a correlation length to a typical change in temperature. Two molecules separated by $45 \AA$ will move apart by approximately $1.5 \AA$ on a temperature increase of $80^{\circ} \mathrm{C}$. A movement of this magnitude is clearly not permitted for the $-\mathrm{CH}_{2}-$ groups near the rigidly fixed head groups. The broadening of the GID peak is therefore due to the frustration between the different thermal responses of the head and tail regions of the hydrocarbon molecules when the monolayer temperature differs from the $\sim 25^{\circ} \mathrm{C}$ monolayer formation temperature. (We anticipate that varying the monolayer formation temperature would result in a shift in the temperature at which the correlation length is maximized. Self-assembly at elevated temperature will be the subject of future studies.) On cooling from room temperature, the order within a correlation length must increase to account for the increase in scattering intensity. As noted above, however, the translational correlation length itself actually decreases.

These thermal changes to the monolayer can be contrasted to similar measurements made of cadmium arachidate LB monolayers on silicon oxide using electron diffraction, ${ }^{14}$ which found an irreversible loss of signal occurring in the temperatures range $50-100^{\circ} \mathrm{C}$. The author surmised that the loss of single molecules from the film at this temperature destroys the in-plane order. In contrast, we found the changes in GID peak intensity with temperature to be quasireversible, with no evidence for a phase transition over the temperature range studied. The different thermal responses of the LB films and alkylsiloxane films are probably a consequence of the very different bonding of the two types of monolayers; LB films are physisorbed on the surface while alkylsiloxane monolayers are chemisorbed to the surface via strong siloxane bonds.

\section{X-ray-induced damage}

Figure 6 shows changes to the GID peak with $x$-ray exposure. During the scans, which took about 9 min each, approximately $7 \times 10^{10}$ photons $\mathrm{s}^{-1} \mathrm{~cm}^{-2}$ fell on the illuminated region of the sample. The sample remained in the beam for approximately $18 \mathrm{~min}$ between scans. The "half life" of the GID peak in this beam was approximately 90 min, during which time about $4 \times 10^{14}$ photons $\mathrm{cm}^{-2}$ fell on the sample. Using the mean alkylsiloxane molecular area calculated from $x$-ray reflectivity, this integrated flux is equivalent to about 1 photon per alkylsiloxane molecule.

During x-ray exposure the HWHM of the GID peak broadens and the position of the peak shifts to slightly lower $q$, indicating that the material giving rise to GID scattering is becoming increasingly disordered and more loosely packed. Previous evidence for $\mathrm{x}$-ray damage of alkylsiloxane films came from changes to the wetting properties (the surface energy increases, reducing the contact angle in the regions exposed to the $\mathrm{x}$-ray beam ${ }^{24}$ ) and from changes to the reflectivity of simple alkylsiloxane monolayers and similar monolayers with a methylene group replaced by a sulfur atom. ${ }^{38}$ Both these manifestations of $x$-ray damage were observed again. We note two possible mechanisms for the observed effect. Photons inelastically scattered within a few tens of angstroms of the surface generate photo- and secondary

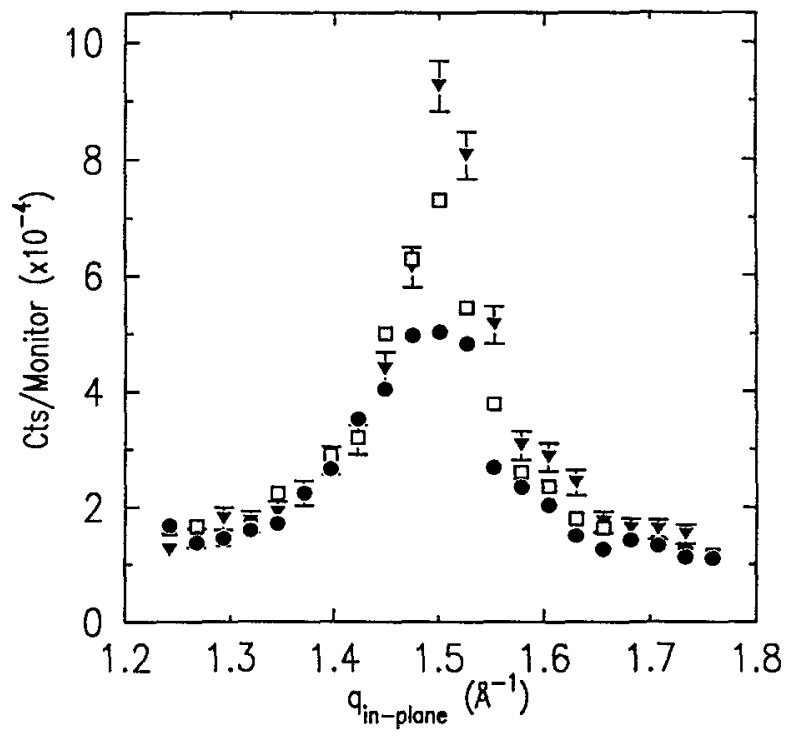

FIG. 6. The evolution of the GID peak with $x$-ray exposure to one region of the sample. Scans, lasting 9 min each, were taken on immediate exposure of the sample $(\nabla)$, after $27 \mathrm{~min}(\square)$, and after $54 \mathrm{~min}(O)$. The sample was left exposed to the beam between scans. For clarity, error bars are shown only for the first data set.

electrons, which in turn have large cross sections for inelastic, and hence damaging, scattering in the monolayer ${ }^{39}$ In addition, free radical production at the surface, by either photons or electrons, can result in a chemical chain reaction that damages more than one alkyl chain per initiating radical. Radical chain reactions are expected to be especially important if oxygen is present in the sample chamber, since hydroperoxides and hydroperoxy radicals might then be formed. The experiments reported here were, however, conducted under helium, presumably limiting the incorporation of oxygen into the monolayer during $\mathrm{x}$-ray exposure. We did not further characterize the damaged regions.

\section{SUMMARY AND CONCLUSIONS}

Using GID and $x$-ray reflection, we have studied the structure of 18 carbon alkylsiloxane monolayers formed by self-assembly from solution onto silicon-oxide-coated silicon wafers. Complete monolayers were found to have shortrange in-plane liquidlike order with translational correlation lengths of about $45 \AA$. There was no evidence of in-plane crystalline structure. These translational correlation lengths are comparable to the lengths found in LB and organosulfur single-chain hydrocarbon monolayers anchored on solid substrates $^{12,15}$ and shorter than the translational correlation lengths found for Langmuir films on water. ${ }^{7-10} \mathrm{~A}$ "cartoon" showing a one-dimensional projection of the in-plane order of a complete monolayer is shown in Fig. 3. The molecules appear to be packed with undistorted hexagonal local order and a mean molecular area of $20.2 \AA^{2}$.

For incomplete monolayers the position of the GID peak is independent of the mean alkylsiloxane molecular area; however, the range and perfection of local order decreases rapidly with increasing mean molecular area. The 
differing packing densities inferred from GID and reflectivity data indicate that the films are inhomogeneous.

For complete monolayers, the shifting of the GID peak with temperature indicates that the material contributing to this peak has a thermal expansion coefficient similar to that of short liquid $n$-alkanes. Either heating or cooling the sample from room temperature decreases the in-plane correlation length. This is presumably a consequence of the tethering of the molecules to a substrate with a negligible thermal-expansion coefficient. This restricts the motion of the alkane chains near the head group, resulting in packing frustration which leads to broadening of the GID peak. Changes in the monolayer structures are quasireversible upon heating, with no indication of loss of material from the monolayer.

Hydrocarbon monolayer films on solid substrates were found to be quite susceptible to $\mathrm{x}$-ray-induced damage.

\section{ACKNOWLEDGMENTS}

We acknowledge the assistance of the X16B staff. This work was supported by the National Science Foundation through Grant No. NSF DMR-89-20490 and by support from the Office of Naval Research and the Defense Advanced Research Projects Agency (DARPA). Research carried out at the National Synchrotron Light Source, Brookhaven National Laboratory, is supported by the Department of Energy, Material Sciences, and Division of Chemical Sciences under Contract No. DE-AC0276CH00016. X16B is supported by AT\&T Bell Laboratories.

'J. D. Swalen et al, Langmuir 3, 932 (1987).

${ }^{2}$ L. DeQuan, M. A. Ratner, T. J. Marks CH. Zhang, J. Yang, and G. K. Wong, J. Am. Chem. Soc. 112, 7389 (1990).

${ }^{3}$ A. J. Nozik, B. R. Tucker, J. A. Turner, and M. W. Peterson, J. Am. Chem. Soc. 110, 763 (1988).

${ }^{4}$ S. R. Wasserman, H. Biebuyck, and G. M. Whitesides, J. Mater. Res. 4, 886 (1989).

${ }^{5}$ D. R. Nelson, in Phase Transitions and Critical Phenomena, edited by C. Domb and J. L. Lebowitz (Academic, London, 1983), Vol. 7.

${ }^{\circ} \mathrm{W}$. F. Brinkman, D. S. Fisher, and D. E. Moncton, Science 217, 693 (1982).

${ }^{7}$ P. Dutta, J. B. Peng, B. Lin, J. B. Ketterson, M. Prakash, P. Georgopoulos, and S. Ehrlich, Phys. Rev. Lett. 58, 2228 (1987).

${ }^{8} \mathrm{~K}$. Kjaer, J. Als-Nielsen, C. A. Helm, L. A. Luxhuber, and H. Mohwald, Phys. Rev. Lett. 58, 2224 (1987).
${ }^{9}$ S. G. Wolf, L. Leiserowitz, M. Lahav, M. Deutsch, K. Kjaer, and J. AlsNielsen, Nature (London) 328, 63 (1987).

${ }^{10}$ M. L. Schlossman, D. K. Schwartz, P. S. Pershan, E. H. Kawamoto, G. J. Kellogg, and S. Lee, Phys. Rev. Lett. 66, 1599 (1991).

${ }^{13}$ M. Seul, P. Eisenberger, and H. M. McConnell, Proc. Natl. Acad. Sci. U.S.A. 80, 5797 (1983).

${ }^{12}$ S. Garoff, H. W. Deckman, J. H. Dunsmuir, M. S. Alvarez, and J. M, Bloch, J. Phys. (Parts) 47, 701 (1986).

${ }^{13}$ V. Vogel and C. Woll, J. Chem. Phys. 84, 5200 (1986).

${ }^{14}$ J. E. Riegler, J. Phys. Chem. 93, 6475 (1989).

${ }^{15}$ L. Strong and G. M. Whitesides, Langmuir 4, 546 (1988).

${ }^{16}$ C. E. D. Chidsey, G.-Y. Liu, P. Rowntree, and G. Scoles, J. Chem. Phys. 91, 4421 (1989).

${ }^{17}$ J. Daillant, J. J. Benattar, and L. Léger, Phys. Rev. A 41, 1963 (1990).

${ }^{18}$ A. J. Kox, J. P. J. Michels, and F. W. Wiegel, Nature (London) 287, 317 (1980).

${ }^{19}$ J. Harris and S. A. Rice, J. Chem. Phys. 88, 1298 (1988).

${ }^{20}$ J. P. Bareman, G. Cardini, and M. L. Klein, Phys. Rev. Lett. 60, 2152 (1988),

${ }^{21}$ J. P. Bareman and M. L. Klein, J. Phys. Chem. 94, 5202 (1990).

${ }^{22}$ A. Ulman, J. E. Eilers, and N. Tillman, Langmuir 5, 1147 (1989).

${ }^{23}$ I. M. Tidswell, B. M. Ocko, P. S. Pershan, S. R. Wasserman, G. M. Whitesides, and J. D. Axe, Phys. Rev. B 41, 1111 (1990).

${ }^{24}$ S. R. Wasserman, G. M. Whitesides, I. M. Tidswell, B. M. Ocko, P. S. Pershan, and J. D. Axe, J. Am. Chem. Soc. 111, 5852 (1989).

${ }^{25}$ L. S. Bartell and J. F. Betts, J. Phys. Chem. 64, 1075 (1960).

${ }^{26}$ R. Maoz and J. Sagiv, J. Colloid Interface Sci. 100, 465 (1984).

${ }^{27}$ S. R. Cohen, R. Naaman, and J. Sagiv, J. Phys. Chem. 90, 3054 (1986).

${ }^{28}$ S. R. Wasserman, Y-T. Tao, and G. M. Whitesides, Langmuir 5, 1074 (1989).

${ }^{29}$ W. C. Marra, P. Eisenberger, and A. Y. Cho, J. Appl. Phys. 50, 6927 (1979).

${ }^{30}$ Failure of this model occurs just above the critical angle for interface rms roughnesses greater than approximately $8 \AA$. For materials where some fraction of the electrons have binding energies greater than the photon energy, the electron density is replaced by an effective electron density which gives less weight to the tightly bound electrons.

${ }^{31}$ J. Als-Nielsen. Physica 140A, 376 (1986).

${ }^{32}$ I. M. Tidswell, T. R. Rabedeau, P. S. Pershan, M. V. Baker, and G. M. Whitesides (unpublished)

${ }^{33}$ Structure of Liquid Crystal Phases, edited by P. S. Pershan (World Scientific, Singapore, 1988).

${ }^{34}$ The two small differences are the diffuse rather than sharp silicon/siliconoxide interface and the lack of structure in the silicon-oxide/hydrocarbon interface. Both of these features can be ascribed to a slightly rougher initial substrate, these features being only poorly characterized.

${ }^{35}$ Physical Constants of Hydrocarbons, edited by G. Egloff (Reinhold, New York, 1939).

${ }^{36} \mathrm{CRC}$ Handbook of Chemistry and Physics, edited by R. C. Weast (CRC, Boca Raton, FL, 1985).

${ }^{37}$ I. M. Tidswell, B. M. Ocko, P. S. Pershan, S. R. Wasserman, G. M. Whitesides, and J. D. Axe (unpublished).

${ }^{38}$ M. V. Baker, I. M. Tidswell, T. A. Rabedeau, P. S. Pershan, and G. M. Whitesides (unpublished).

${ }^{39}$ P. E. Laibinis, R. L. Graham, H. A. Biebuyck, and G. M. Whitesides (unpublished). 